\title{
Prevenção do estresse oxidativo na síndrome de isquemia e reperfusão renal em ratos com suplementação nutricional com antioxidantes
}

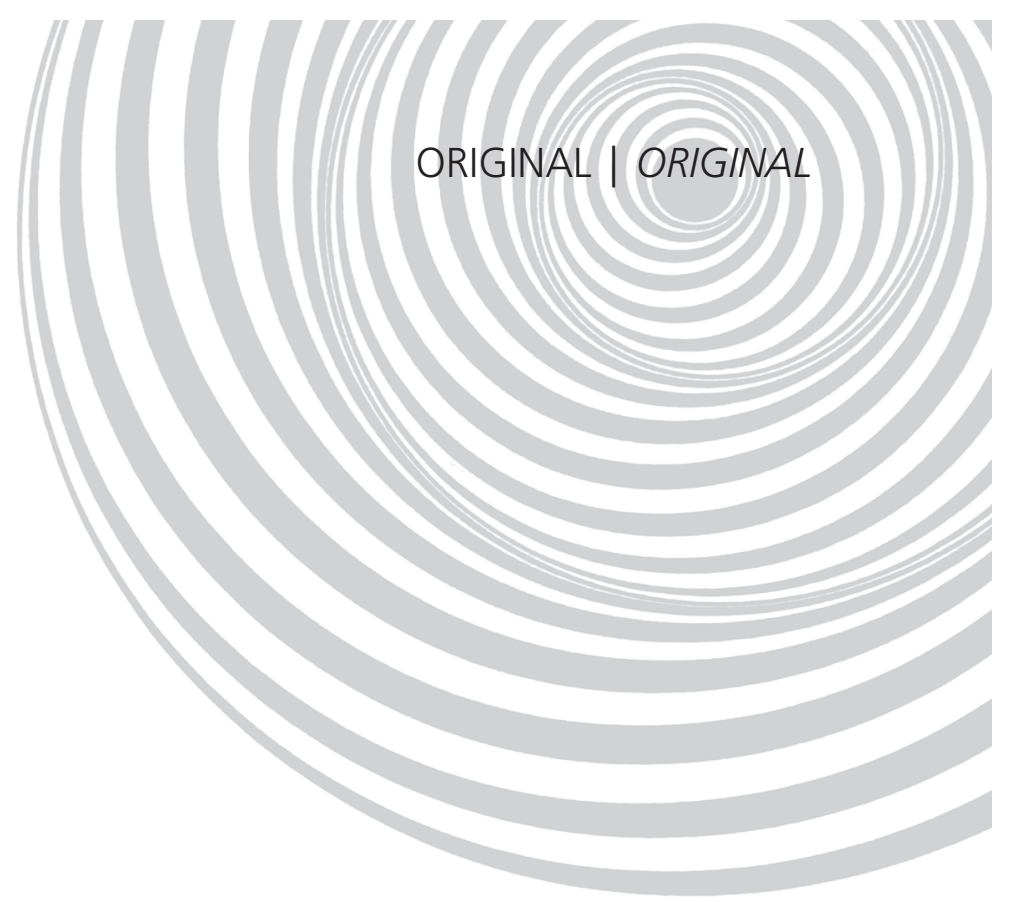

\author{
Prevention of oxidative stress in renal \\ ischemia-reperfusion syndrome in rats \\ with nutritional antioxidant \\ supplementation
}

Sandro PERCÁRIO'

RES U M O

\section{Objetivo}

Verificar o potencial efeito protetor de suplementação com vitaminas antioxidantes em um modelo de síndrome de isquemia-reperfusão renal em ratos.

\section{Métodos}

Foram utilizados 29 ratos Wistar, divididos em três grupos: Grupo I e II ( $\mathrm{n}=10$ cada), submetidos a indução do estresse oxidativo pela aplicação de 60 minutos de isquemia renal, seguidos de 10 minutos de reperfusão; adicionalmente, os animais do Grupo II foram pré-tratados por doze dias com vitaminas antioxidantes (vitamina C $11,43 \mathrm{mg} / \mathrm{kg}$ e vitamina E 28,57mg/kg) antes da submissão à isquemia; Grupo III ( $\mathrm{n}=9$ ), correspondendo aos animais Sham, que foram manipulados de forma equivalente aos outros grupos, porém sem indução do estresse oxidativo e sem suplementação antioxidante. Findo isso, as amostras de sangue e os rins foram colhidos para avaliação dos níveis do malondialdeído, do ácido úrico e da capacidade antioxidante total.

\section{Resultados}

Para o malondialdeído e ácido úrico do Grupo I foi observado um aumento estatisticamente significante $(p<0,01)$ em relação ao Grupo III, o qual não apresentou diferença em relação ao Grupo II. Para os níveis de capacidade antioxidante total, foi encontrada uma diminuição nos animais do Grupo I em relação aos Grupos II e III $(p<0,01)$.

\footnotetext{
1 Universidade Federal do Pará, Núcleo de Medicina Tropical, Laboratório de Estresse Oxidativo. Av. Generalíssimo Deodoro, 92,66055-240, Belém, PA, Brasil. E-mail: <percario@ufpa.br>.
} 
260 | S. PERCÁRIO

\section{Conclusão}

Esses dados confirmam não apenas a efetiva participação do estresse oxidativo neste modelo de síndrome de isquemia e reperfusão renal em ratos, como também que o uso de vitaminas antioxidantes, associadas à dieta, pode proteger os animais das alterações oxidativas.

Termos de indexação: Antioxidantes. Estresse oxidativo. Radicais livres. Síndrome de esquemia. Transplante de rins. Traumatismo por reperfusão. Vitaminas.

\section{A B S T R A C T}

\section{Objective}

The objective of this study was to verify the potential protective effect of antioxidant vitamin supplementation in a model of renal ischemic-reperfusion injury in rats.

\section{Methods}

Twenty-nine Wistar rats were divided into three groups: groups I and II ( $n=10$ each), were submitted to 60 minutes of renal ischemia, followed by 10 minutes of reperfusion; additionally, animals of group II were treated for twelve days with antioxidant vitamins (vitamin C $11.43 \mathrm{mg} / \mathrm{kg}$ and vitamin E $28.5 \mathrm{mg} / \mathrm{kg}$ ) before being submitted to ischemia; In group III (n=9), the animals were treated like the other groups but not submitted to ischemic-reperfusion injury and not given antioxidant supplements. Subsequently, blood samples and the kidneys were collected for assessment of malondialdehyde, uric acid and total antioxidant capacity.

\section{Results}

The malondialdehyde and uric acid of group I was significantly higher than those of group III $(p<0.01)$, which in turn did not differ from group II. The levels of total antioxidant capacity of the animals of group I was lower than those of groups II and III $(p<0.01)$.

\section{Conclusion}

These data confirmed the effective participation of oxidative stress in this model of renal ischemia-reperfusion syndrome in rats and showed that the use of antioxidant supplementation can protect the animals from oxidative changes.

Indexing terms: Antioxidants. Oxidative stress. Free radicals. Reperfusion injury. Vitamins.

\section{N T R O D U Ç Ã O}

O transplante renal é o transplante de órgão vascularizado realizado com maior frequência, há mais tempo e do qual se tem maior experiência no Brasil'. É o tratamento de escolha para os pacientes com insuficiência renal crônica terminal que não apresentam contraindicação por ser, das formas de substituição da função renal, a que oferece a melhor reabilitação com o menor custo social ${ }^{2-4}$.

Pacientes aguardando novos rins são mantidos em hemodiálise, o que pode causar alterações oxidativas pela ativação de fagócitos e pela depleção de antioxidantes plasmáticos ${ }^{5}$.

Apesar dos recentes conhecimentos da sua base molecular ${ }^{6}$ a rejeição é um dos maiores problemas do transplante renal. Esforços consideráveis têm sido feitos para correlacionar a morfologia do enxerto com a evolução clínica da rejeição. Entretanto, estudos histológicos de uma biópsia renal nunca conseguem fornecer mais do que uma "foto instantânea" e com um foco limitado de um processo sistêmico complexo e de evolução contínua, que também está sendo modificado pela imunossupressão?

Na rejeição renal, frequentemente o órgão é infiltrado por células fagocitárias. Assim é possível que as Espécies Reativas Tóxicas do Oxigênio (ERO), produzidas por essas células ${ }^{5}$, contribuam para a disfunção do órgão por meio do processo chamado "estresse oxidativo" $5,8,9$.

Adicionalmente, o processo isquêmico no rim é caracterizado por diminuição do fluxo 
sanguíneo renal, decréscimo da filtração glomerular e diminuição do coeficiente de ultrafiltração renal, além da disfunção tubular secundária à obstrução e dano do epitélio tubular ${ }^{10}$. A conjunção desses fatores oferece as condições necessárias para a instalação da Síndrome de Isquemia-Reperfusão (SIR), que é uma causa importante da disfunção orgânica em transplante renal ${ }^{11,12}$.

Os mecanismos propostos para explicar a lesão secundária à SIR são diversos: liberação de ERO durante a reoxigenação tecidual; acumulação leucocitária; e subsequente liberação de ERO adicionais e enzimas líticas ${ }^{11}$.

A SIR é um mecanismo importante de dano oxidativo, na qual a hipóxia cria condições ideais para a produção de radicais livres após a reoxigenação. O tempo de isquemia e a intensidade da reoxigenação determinarão o grau de lesão, comprometendo principalmente a membrana celular; porém seus efeitos lesivos também podem acontecer ao nível dos ácidos nucléicos, assim como das proteínas e outros constituintes da célula ${ }^{5}$.

O mecanismo de ação dos fenômenos que se desenvolvem na SIR está exposto na Figura 1 e inicia-se com a instalação de um processo isquêmico, com interrupção da fosforilação oxidativa e, consequentemente, redução a níveis mínimos da quantidade de Adenosina Trifosfato (ATP). Diante dessa condição, para a manutenção do metabolismo basal da célula, o ATP de reserva celular é degradado em Adenosina Difosfato (ADP) e Adenosina Monofosfato (AMP), transformando-se esse último, consecutivamente, em adenosina, inosina e hipoxantina. Os nucleotídeos (ATP, ADP, AMP) são impermeáveis às células, enquanto os nucleosídeos (adenosina e inosina) e a base hipoxantina são permeáveis, saindo da célula e esgotando suas reservas. No período de reperfusão, durante a reoxigenação, quando deveria haver ressíntese do ATP, a depleção celular dessas últimas substâncias impede esse processo ${ }^{13}$.

Paralelamente, existe um sistema enzimático constituído pela xantina-desidrogenase e xantina-oxidase, envolvido neste processo. Por

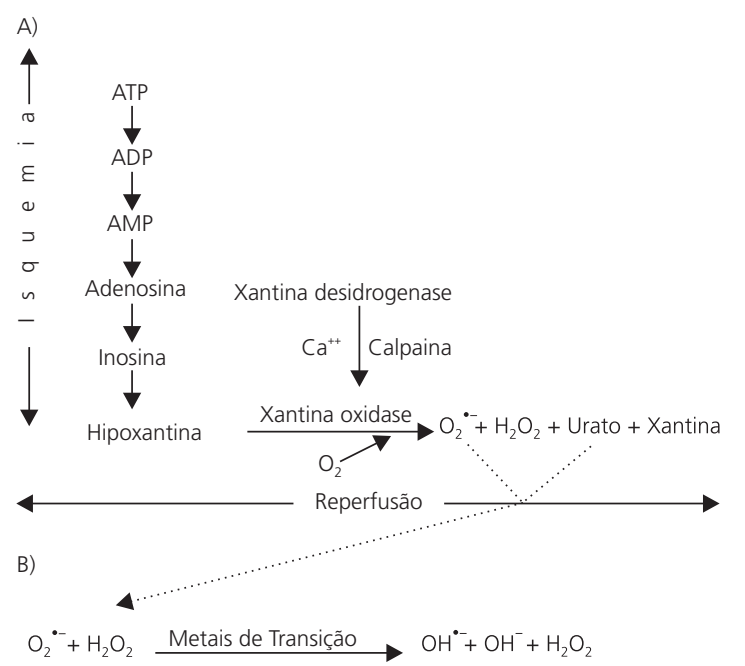

Figura 1. Mecanismo da síndrome de isquemia-reperfusão ${ }^{14}$. Nota: ADP: Adenosina difostato; ATP: Adenosina trifosfato; AMP: Adenosina monofosfato.

ocasião da isquemia, devido à descompartimentalização do cálcio $\left(\mathrm{Ca}^{++}\right)$intracelular, ocorre ativação de proteases, as quais convertem a xantina-desidrogenase em xantina-oxidase. Essa última enzima, durante a reperfusão, transforma a hipoxantina em ácido úrico e nesse processo são gerados radicais ânion superóxido $\left(\mathrm{O}_{2}{ }^{*}\right)$ e o peróxido de hidrogênio $\left(\mathrm{H}_{2} \mathrm{O}_{2}\right)^{13}$. Esses últimos se combinam na reação de Haber-Weiss para produzir o radical livre hidroxila $\left(\mathrm{OH}^{*}\right)$, potencializando o dano oxidativo, uma vez que esse radical é cerca de 1 milhão de vezes mais reativo que seus precursores (Figura 1) ${ }^{14}$.

Os radicais $\mathrm{O}_{2}{ }^{--}$e seus produtos de redução, o $\mathrm{H}_{2} \mathrm{O}_{2}$ e, principalmente o radical $\mathrm{OH}^{\circ}$, são alguns dos responsáveis pela lesão celular, promovendo a peroxidação lipídica, com lesão das mitocôndrias, lisossomos e da própria membrana celular, levando à morte das células. No rim, tais alterações são responsáveis por um aumento da permeabilidade tubular com perda das funções de transporte, bem como redução da fosforilação oxidativa mitocondrial e liberação inapropriada de enzimas líticas lisossômicas, que acelerariam o processo de degradação celular, com consequente diminuição da função renal ${ }^{10-11}$. 
Aliado ao dano direto, agravando o processo, o aumento precoce da produção de radicais livres na isquemia-reperfusão compromete seletivamente a transdução do sinal para a via da produção e liberação do NO. A pequena quantidade de óxido nítrico (NO) produzida reage rapidamente na presença de altas concentrações de $\mathrm{O}_{2}{ }^{*-}$ formando o radical livre peroxinitrito (ONOO*). Na ausência de NO, plaquetas e neutrófilos aderem à superfície da célula endotelial, que se torna ativada e libera fatores vasoativos (serotonina, ADP, tromboxane, etc.), enzimas hidrolíticas (elastase e colagenase) e citocinas ${ }^{15}$. Isso resulta em lesão endotelial adicional e acelera o recrutamento e ativação de neutrófilos e plaquetas adicionais. As plaquetas e neutrófilos acumulados ocluem o vaso e o tecido dependente que se torna, uma vez mais isquêmico, gerando um ciclo vicioso de produção de radicais livres ${ }^{16}$.

Algumas evidências, como a geração de produtos de peroxidação lipídica, possivelmente derivados da ação dos radicais livres na membrana lipídica, a proteção de lesão isquêmica por substâncias varredoras (sequestradoras) de radicais livres como a enzima Superóxido Dismutase (SOD), glutationa reduzida, vitamina $E$, desferoxamina, dimetiluréia, manitol, bem como a mimetização de alguns aspectos da lesão isquêmica em organelas renais expostas a radicais livres, reforçam a sugestão do envolvimento de radicais livres na lesão isquêmica renal 17-19.

Os mecanismos da injúria isquêmica são comuns para todos os órgãos sólidos, mas existem algumas características específicas para cada órgão. As consequências da injúria de isquemia-reperfusão dependem da duração da isquemia, temperatura e da natureza do órgão ${ }^{12}$.

A insuficiência renal assume importância no transplante renal. O processo de captação e preservação do órgão determina graus variáveis de isquemia do rim a ser transplantado, o que traz consequências deletérias, tais como uma menor sobrevida e comprometimento da função do órgão, após ser transplantado. Dessa forma, inevitavelmente, todo enxerto de órgão sólido é subme- tido a algum grau de injúria tissular decorrente da isquemia-reperfusão ${ }^{20}$.

Segundo Southard et al. ${ }^{21}$, dentre as medidas preventivas dessa lesão, está a composição das diferentes soluções de preservação do órgão. Esses mesmos pesquisadores introduziram uma solução de preservação que tem atraído muito interesse por ser tão ou mais eficaz para a preservação renal do que outras. Ela difere fundamentalmente das outras pela presença dos antioxidantes alopurinol e glutationa.

A SIR é um fenômeno complexo, que contribui para a mortalidade e morbidade dos transplantes clínicos de órgãos sólidos. Embora a introdução de melhores soluções de preservação na prática clínica tenha reduzido a severidade das lesões isquêmicas, a injúria da isquemia-reperfusão ainda permanece como um dos maiores problemas dos transplantes ${ }^{22}$. Várias estratégias e recursos são utilizados pelas células para prevenir ou diminuir a injúria celular causada pelo estresse oxidativo. Esses mecanismos de defesa antioxidantes celulares têm por finalidade prevenir a formação de radicais livres, converter espécies oxidantes tóxicas em menos tóxicas, preservar a compartimentalização celular - que é vital para as estruturas celulares - ou promover o reparo do dano causado pelos radicais livres ${ }^{23}$. Dessa forma, é possível que a suplementação nutricional com substâncias antioxidantes possa minimizar o dano oxidativo decorrente da SIR em indivíduos submetidos a transplantes renais.

Diante do exposto, este estudo pretendeu verificar o potencial efeito protetor de dieta rica em vitaminas antioxidantes em um modelo de isquemia e reperfusão renal aguda em ratos.

\section{MÉ TO D O S}

Foram utilizados 29 ratos Wistar, machos, adultos, com pesos entre 200 e $250 \mathrm{~g}$, os quais foram divididos em três grupos:

- Grupo I ( $n=10)$, submetidos a 60 minutos de isquemia renal, seguidos de 10 minutos de reperfusão; 
- Grupo II ( $n=10)$, no qual os animais foram pré-tratados por 12 dias com antioxidantes antes da submissão ao período de 60 minutos de isquemia renal e 10 minutos de reperfusão;

- Grupo III ( $n=9$ ), é o grupo Sham, no qual os animais foram submetidos à eutanásia imediatamente após o período de manipulação equivalente ao grupo submetido à reperfusão aguda.

Os animais foram mantidos em biotério durante o período do experimento, com ração (Purina Labina; Nestlé Purina PetCare Company) e água ad libitum, controle de ruídos e da temperatura ambiente, ciclo escuro-claro de $12 \mathrm{~h}$ cada, atendendo-se às observações preconizadas pelo Colégio Brasileiro de Experimentação Animal (COBEA). O estudo foi autorizado pela Comissão de Ética em Pesquisa da Fundação UNIRG, conforme parecer emitido em 22 de novembro de 2005.

Para administração da solução de vitaminas, os animais do Grupo II foram pré-tratados com vitamina $C$ associada ao Trolox, um análogo sin-tético da vitamina E. Essas vitaminas foram diluídas em água destilada nas seguintes doses: $28,57 \mathrm{mg} / \mathrm{kg}$ peso do animal de vitamina C ( $L$ ascorbic acid; aldrich A92902), e 6,64mg/kg peso do animal de Trolox (Aldrich 238813), equivalente a 11,43mg/kg de vitamina E. Foi utilizado o Trolox, análogo hidrossolúvel da vitamina $\mathrm{E}$, com finalidade de permitir a solubilização e a administração conjunta das vitaminas aos animais por gavagem. Os níveis dessas vitaminas presentes na ração oferecida aos animais eram desprezíveis, conforme informação oferecida pelo seu fabricante (vitamina E $82,0 \mathrm{mg} / \mathrm{kg}$ de ração e vitamina $C<0,5 \mathrm{mg} / \mathrm{kg}$ de ração). Optou-se pela suplementação pelo período de doze dias anteriores à indução do estresse oxidativo nos animais, de forma a garantir não apenas que as eventuais diferenças nos níveis desses antioxidantes entre os animais fossem minimizadas, bem como que houvesse um nível elevado desses antioxidantes no momento da ocorrência da SIR. A suplementação com antioxidantes (vitamina C e Trolox) foi realizada com base na dose terapêutica utilizada em humanos ( $2 \mathrm{~g}$ de vitamina $\mathrm{C}$ e $800 \mathrm{mg}$ de vitamina $\mathrm{E}$ diárias para um indivíduo de $70 \mathrm{~kg}$ ), administrada de forma proporcional ao peso dos animais e diretamente ao estômago dos animais (gavagem), de forma a se assemelhar aos procedimentos terapêuticos aplicados em humanos.

No indução da isquemia renal, os animais foram submetidos à anestesia com hidrato de cloral (400 $\mathrm{mg} / \mathrm{kg}$ peso; i.p.). Foi realizada uma laparotomia xifo-umbilical para exposição da cavidade peritoneal, sendo afastadas as vísceras móveis para exposição dos vasos renais, os quais foram dissecados, permitindo a nefrectomia do rim contra lateral e, após isso, as vísceras foram afastadas para a exposição do rim esquerdo, onde foi feita a dissecação dos vasos renais, o isolamento da artéria renal esquerda e a colocação de uma presilha metálica nessa artéria. Após o período de 60 minutos, a presilha foi retirada e o animal foi submetido à reperfusão de 10 minutos, sendo posteriormente submetido à eutanásia e a coleta de amostras de sangue por punção intracardíaca. Adicionalmente, os rins esquerdos dos animais dos Grupos I e II foram retirados.

Os animais do Grupo III foram submetidos à exposição da cavidade peritoneal, seguida da retirada do rim esquerdo e coleta de amostra de sangue, após o período de manipulação equivalente aos grupos submetidos à isquemia e reperfusão aguda, sem que houvesse a isquemia da artéria renal esquerda.

Foram avaliados os níveis plasmáticos e renais do Malondialdeido (MDA), que é um indicador da peroxidação lipídica, do Ácido Úrico (AU) e níveis plasmáticos da Capacidade Antioxidante Total (TAC). forma:

O preparo das amostras deu-se da seguinte

- Plasma: foi obtido pela centrifugação do sangue total coletado com heparina sódica em 2500rpm por 15 minutos.

- Homogeneizado de rim: os rins foram pesados e colocados em solução tampão salina fosfato PBS, na proporção 1:10 (m:m), dentro do béquer em que foram pesados. Em seguida, os rins foram homogeneizados por um disrruptor de células ultrassônico. 
A dosagem do MDA foi realizada segundo o método de Khon \& Liversedge ${ }^{24}$, modificado por Percário et al. ${ }^{25}$.

A capacidade antioxidante total foi avaliada utilizando o Kit Total Antioxidant Status (Randox Laboratories Ltd., NX2332), e a dosagem de ácido úrico foi realizada utilizando o Kit Ácido Úrico UOD-ANA (Labtest, Cat. 51-4/30).

Após a verificação de eventuais pontos discrepantes pelo método do intervalo interquartil, foi feita uma análise estatística a fim de comparar os grupos ao grupo controle, utilizando o teste t-Student não paramétrico, além do uso do teste t-Student paramétrico para comparação intragrupo. Foi considerado um nível de significância de $5 \%(p \leq 0,05)$ para todas as análises.

\section{RES ULTADOS}

Os valores de MDA, TAC e AU estão apresentados na Tabela 1. Para as amostras plasmáticas não houve diferenças significativas entre os grupos para o MDA. Para as amostras renais do Grupo I foi observado um aumento $(p<0,01)$ em relação ao Grupo III. Já para o Grupo II, não houve diferença estatisticamente significante em relação ao Grupo III, porém é menor que o Grupo I $(p<0,01)$.

Para os níveis plasmáticos de TAC foi encontrada uma diminuição nos animais do Grupo I em relação ao Grupo III $(p<0,01)$ e em relação ao Grupo II não houve diferença estatisticamente significante em relação ao Grupo III.

Para o AU foi observado um comportamento semelhante ao do MDA, onde não houve diferença estatisticamente significante entre os grupos para amostras plasmáticas, enquanto para os níveis renais, no Grupo I, foi observado um aumento estatisticamente significante em comparação ao Grupo III $(p<0,01)$, e no Grupo II não foi observada diferença estatisticamente significante em relação ao Grupo III, porém é estatisticamente menor que o Grupo I $(p<0,01)$.

\section{DISCUSS ÃO}

O transplante renal é realizado com bastante frequência e é um tratamento que pode aumentar as chances de sobrevida de pacientes que necessitam de novos órgãos. Uma vez que existe forte sugestão do envolvimento dos radicais livres nos mecanismos de rejeição de enxertos renais, a realização de estudos com suplementação de antioxidantes poderá contribuir para elucidar os mecanismos da rejeição do órgão pelo receptor, podendo contribuir para o desenvolvimento de técnicas de transplantes capazes de aumentar as taxas de sobrevida. Dessa forma, estudamos um modelo animal de síndrome de isquemia e reperfusão renal, a qual é um mecanismo importante associado ao transplante renal em que ocorre maciça produção de radicais livres.

As dosagens foram realizadas no plasma e no rim dos animais para verificar a repercussão à distância do dano causado pela síndrome de isquemia e reperfusão, com vistas a verificar uma eventual possibilidade prognóstica das dosagens plasmáticas em humanos. No entanto os valores de MDA e AU não diferiram entre os grupos para as amostras plasmáticas, comportamento diverso

Tabela 1. Valores dos níveis plasmáticos e renais para malondialdeído, capacidade antioxidante total e ácido úrico. Gurupi (TO), 2006.

\begin{tabular}{|c|c|c|c|c|c|c|c|c|c|c|c|}
\hline \multirow{3}{*}{ Grupo } & \multirow[b]{3}{*}{$n$} & \multicolumn{4}{|c|}{ MDA (ng/mL) } & \multirow{2}{*}{\multicolumn{2}{|c|}{$\frac{\text { TAC (mmol/L) }}{\text { Plasma }}$}} & \multicolumn{4}{|c|}{$\mathrm{AU}(\mathrm{mg} / \mathrm{dL})$} \\
\hline & & \multicolumn{2}{|c|}{ Plasma } & \multicolumn{2}{|c|}{ Rim } & & & \multicolumn{2}{|c|}{ Plasma } & \multicolumn{2}{|c|}{ Rim } \\
\hline & & $M$ & DP & M & DP & M & DP & $\mathrm{M}$ & DP & $M$ & DP \\
\hline I (Isquemia) & 10 & 1211,0 & 790,0 & 2749,0 & $358,0^{b}$ & 0,9 & $0,4^{\text {b }}$ & 2,7 & 0,9 & 13,0 & $1,5^{b}$ \\
\hline II (Vitamina) & 10 & 844,0 & $791,0^{a}$ & 1755,0 & $409,0^{c, d}$ & 1,4 & $0,3^{c, d}$ & 2,6 & $1,0^{\mathrm{a}}$ & 10,8 & $1,5^{c, d}$ \\
\hline III (Sham) & 9 & 857,0 & $879,0^{a}$ & 1178,0 & $776,0^{c}$ & 1,5 & $0,3^{c, d}$ & 2,5 & $0,4^{\mathrm{a}}$ & 9,6 & $1,6^{c}$ \\
\hline
\end{tabular}

a. não significante x Grupo I; b: p<0,01 x Grupo III; c: p<0,01 x Grupo I; d. não significante x Grupo III.

MDA: malondialdeído; PAC: capacidade antioxidante total; AU: ácido úrico; M: média; DP: desvio-padrão. 
daquele apresentado pelas amostras de tecido renal. Esse fato sugere que não ocorra repercussão sistêmica das alterações locais nesse modelo, ou ainda que a grande variabilidade de comportamentos apresentados individualmente pelos animais estudados possa ter mascarado esta repercussão.

Paralelamente, o emprego de método para avaliação laboratorial de MDA por método fotométrico sem separação preparativa por Cromatografia Líquida de Alta Performance (HPLC) pode ter comprometido a análise dos resultados em amostras de plasma, em virtude de sua baixa especificidade. Para as amostras de tecido renal, as diferenças encontradas entre os grupos sugerem que o emprego do método tenha sido adequado, a despeito de sua baixa especificidade.

Realizou-se a dosagem de AU para verificar se a produção de radicais livres decorria da síndrome de isquemia e reperfusão, uma vez que o AU é subproduto dessa síndrome. Dessa forma, os valores significantemente elevados de AU no tecido renal apresentados pelos animais do Grupo I confirmam que a produção de radicais livres possa ser consequência da síndrome de isquemia e reperfusão, uma vez que o AU é um dos subprodutos da SIR.

Em relação às dosagens de MDA, não foram visualizadas diferenças entre os grupos para as amostras de plasma. Por outro lado, nas amostras de tecido renal, no Grupo I pôde-se observar um aumento significativo em relação ao Grupo III, sugerindo que o processo isquêmico-reperfusional ao qual os animais do Grupo I foram submetidos resultou, de fato, na elevação dos níveis de radicais livres e, consequentemente, no envolvimento do estresse oxidativo. Para o Grupo II, os valores não apresentaram diferenças estatísticas em relação ao Grupo III, sugerindo que a suplementação de vitaminas antioxidantes tenha reduzido o estresse oxidativo nesses animais.

Por outro lado, para visualizar o comportamento das defesas antioxidantes existentes nos animais frente à agressão oxidativa imposta pela síndrome de isquemia e reperfusão, realizaram- -se as dosagens de TAC. Os valores de TAC encontrados nas amostras provenientes de animais do grupo submetido à isquemia-reperfusão (Grupo I) eram menores que os apresentados pelo Grupo III, sugerindo que houve o consumo das defesas antioxidantes frente à agressão oxidativa. A suplementação dos animais com vitaminas antioxidantes (Grupo II), entretanto, parece ter protegido o organismo do estresse oxidativo consequente do processo de isquemia-reperfusão ao qual os ratos foram submetidos, uma vez que a capacidade antioxidante do plasma não sofreu redução, mantendo-se nos níveis dos animais do grupo não submetido à reperfusão, o Grupo III.

Analisando-se coletivamente os resultados de MDA e AU obtidos nas amostras de tecido renal, observa-se um comportamento congruente para os grupos analisados, com aumento de ambos os parâmetros nas amostras oriundas do Grupo I em relação aos outros dois grupos que não apresentaram diferenças entre si. Esse dado era esperado, uma vez que o aumento dos níveis de AU confirma a participação da síndrome de isquemia e reperfusão no processo e, consequentemente, a produção de radicais livres, os quais são capazes de causar peroxidação lipídica e o aumento dos níveis de MDA. Por outro lado, apesar da não existência de diferenças para esses parâmetros nas amostras plasmáticas, ocorreu diminuição do TAC no plasma dos animais submetidos à isquemia e não suplementados (Grupo I), demonstrando o consumo das defesas antioxidantes sistêmicas para mitigar o dano oxidativo imposto pela síndrome de isquemia e reperfusão aos animais. Esse foi o único parâmetro analisado que apresentou repercussão sistêmica, de forma que sua análise em indivíduos transplantados poderia constituir-se em avaliação preditiva de risco para rejeição do enxerto. Entretanto são requeridos mais estudos para confirmação dessa sugestão.

Os dados apresentados neste estudo estão em concordância com a grande maioria dos estudos apresentados na literatura, nos quais o uso de antioxidantes preveniu as alterações oxidativas em modelos experimentais de síndrome de 
isquemia e reperfusão renal. Nesse sentido, em ratos submetidos à SIR renal, o uso dos antioxidantes silimarina ${ }^{26}$, beta-glucana ${ }^{27}$, proantocianidina ${ }^{28}$, melatonina ${ }^{29}$, ácido ascórbio ${ }^{30,31}$, alfa tocoferol ${ }^{29,32,33}$ e resveratro| ${ }^{34}$ promoveram diminuição dos níveis renais de MDA, bem como aumento de marcadores da defesa antioxidante, tais como a glutationa reduzida (GSH). O mesmo comportamento foi observado em modelos de SIR renal em coelhos ${ }^{30,35}$ e em cães ${ }^{36}$.

Por outro lado, alguns estudos apontam que o uso de antioxidantes não promove alterações nos marcadores oxidativos, questionando o benefício da suplementação antioxidante em modelos experimentais da SIR. Nesse caso enquadra-se o estudo de Kontscher et al. ${ }^{37}$ que não encontrou diferenças nos níveis plasmáticos de MDA, TAC ou GSH em porcos suplementados com ácido ascórbico, alfa tocoferol, $\mathrm{N}$-acetilcisteína e selênio e submetidos a transplante renal. Acreditamos que esses resultados possam decorrer de dois fatores que contribuíram para o possível mascaramento dos resultados: a quantidade administrada dos antioxidantes ter sido insuficiente para promover alterações detectáveis, uma vez que foram empregadas doses de apenas $2,5 \mathrm{mg} / \mathrm{kg}$ de peso do animal de alfa tocoferol, bastante inferiores às empregadas no presente estudo, ou ao fato de as análises terem sido realizadas exclusivamente em amostras plasmáticas e, portanto, não refletindo as alterações locais do tecido renal.

Diante do exposto, este estudo reforça a sugestão do potencial benefício da suplementação antioxidante em pacientes a serem submetidos a transplantes; no entanto estudos adicionais são necessários para o entendimento das vias bioquímicas oxidativas envolvidas, notadamente o papel do óxido nítrico, podendo contribuir com a melhoria da qualidade de vida dos pacientes submetidos a transplantes renais.

Esses achados confirmam a efetiva participação do estresse oxidativo neste modelo de síndrome de isquemia e reperfusão renal em ratos e que o uso de antioxidantes associados à dieta pode proteger os animais das alterações oxidativas associadas.

\section{REFERÊ N CIAS}

1. Bambirra EA, Paulino UHM, Tafuri WL, Pereira FEL, Bogliolo L. Noções de imunopatologia. In: Brasileiro Filho G, Pereira EL, Pitella JEH, Bambirra EA, Barbosa AJF, editores. Patologia geral. Rio de Janeiro: Guanabara Koogan; 1993.

2. Manfro RC, Gonçalves LFS. Monitorização do transplante renal. In: Cruz J, Barros RT, Sesso RCC, David Neto E, Suassuna JHR, Heilberg IP, et al., editores. Atualidades em nefrologia. $3^{a}$ ed. São Paulo: Sarvier; 1994.

3. Goldani JC, Bruno RM, Messias AA, Losekann A. Seleção, avaliação e preparo do receptor. In: Neumann J, Abbud Filho M, Garcia, UO. Transplante de órgãos e tecidos. São Paulo: Sarvier; 1997.

4. Bakker CF, Naji A, Markmann JF, Brayman KL. Transplante renal. In: Bakker CF, Naji A, Markmann $\mathrm{JF}$, Brayman K. As Bases biológicas da prática cirúrgica moderna. $15^{a}$ ed. Rio de Janeiro: Guanabara Koogan; 1999.

5. Halliwell B, Gutteridge JMC. Free radicals in biology and medicine. $3^{\text {rd }}$ ed. Oxford: Clarendon Press; 1999.

6. Dedeoglu O, Feld LG. Decreased urinary excretion of nitric oxide in acute rejection episodes in pediatric renal allograft recipients. Ann Meet Transplant Physicians. 1996; 62(12):1936-8.

7. Curtis JJ. Tratamento da insuficiência renal. In: Benetti JC, Plum F editores. Tratado de medicina interna. 20a ed. Rio de Janeiro: Guanabara Koogan; 1997.

8. Mcgratht LT, Treacy R, McClean E, Brown JH. Oxidative stress in cyclosporin and azathioprine treated renal transplant patients. Clin Chimica Acta. 1997; 264(1):1-12. doi: 10.1016/S0009-8981 (97) 00088-0.

9. Fellstrom B. Nonimmune factors for chronic renal allograft dysfunction. Transplantation. 2001; 71 (11 Suppl.):S10-S16.

10. Ernani LR, Teloken C, Souto CAV, Rhoden C, Lucas $\mathrm{ML}$, Bello-Klein A. Effects of L-arginine and L-name on renal ischemia-reperfusion in rats. Braz J Urol. 2001; 27(1):78-83.

11. Boneventre JV. Mechanisms of ischemic acute renal failure. Kidney Int. 1993; 43(5):1160-78. doi: 10.1038/kl.1993.163.

12. Duska D, Hoff U, Park JK, Qun Y, Schneider W, Luft $F C$, et al. Ischemia-reperfusion injury in renal transplantation is independent of the immunologic background. Kidney Int. 2000; 58(5):2166-177. doi: 10.111/j.1523-1755.2000.00390-x.

13. Seguro AC. Lesão celular na isquemia renal. In: Cruz J, Barros RT, Sesso RCC, David Neto E, 
Suassuna JHR, Heilberg IP, et al., editores. Atualidades em nefrologia $3^{a}$ ed. São Paulo: Sarvier; 1994.

14. Percário S. Alterações oxidativas e da defesa antioxidante no broncoespasmo agudo induzido em cobaias [tese]. São Paulo: Universidade Federal de São Paulo; 2000.

15. Moncada S, Palmer RMJ, Higgs EA. Nitric oxide: physiology, pathophysiology, and pharmacology. Pharmacol Rev. 1991; 43(2):109-42.

16. Evora PRB, Pearson PJ, Seccombe JF, Schaft HV. Lesão de isquemia-reperfusão: aspectos fisiopatológicos e a importância da função endotelial. Arq Bras Cardiol. 1996; 66(4):239.

17. Nogueira Jr A, Santos OR. Patogenia e clinica das afecções renais. In: Nogueira Jr A Santos OR. Doença dos rins. São Paulo: Fundo Editorial BYK; 1998.

18. Land W, Zweier JL. Prevention of reperfusioninduced, free radical-mediated acute endothelial injury by superoxide dismutases as an effective tool to delay/prevent chronic allograft failure: a review. Transplant Proc. 1997; 29(6):2567-8. doi: 10.1016/S0 041-1345(97)00509-5.

19. Laranja SMR, Boim MA, Schor N. IRA Isquêmica: Necrose tubular aguda In: Boim MA, Santos OFP, Schor N. Insuficiência renal aguda: fisiopatologia, clínica e tratamento. São Paulo: Sarvier; 1997.

20. Azuma H, Nadeau K, Takad M, Mackenzie HS, Tilney NL. Cellular and molecular predictors of chronic renal dysfunction after initial ischemia/reperfusion injury of a single kidney. Transplantation. 1997; 64(2):190-7.

21. Southard JH, Van TMG, Ametani MS, Vreugdenhil PK, Lindell SL, Pienaar BL, et al. Important components of the UW solution. Transplantation. 1990; 49(2):251-7.

22. Torras J, Cruzado JM, Grinyo JM. Ischemia and reperfusion injury in transplantation. Transplant Proc. 1999; 31(6):2217-8. doi: 10.1016/S0041-1345 (99)00312-7.

23. Shah SU. The role of reactive oxygen metabolites in glomerular disease. Annu Rev Physiol. 1995; 57:245-62. doi: 10.1146/annurev.ph.57.03019 5.001333.

24. Kohn HI, Liversedge M. On a new aerobic metabolite whose production by brain is inhibited by apomorphine, emetine, ergotamine, epinephrine, and menadione. J Pharmacol Experimen Ther. 1944; 82(3):292-300.

25. Percário S, Vital ACC, Jablonka F. Dosagem do malondialdeido. Newslab. 1994; 2(6):46-50.

26. Turgut F, Bayrak O, Catal F, Bayrak R, Atmaca AF, Koc $A$, et al. Antioxidant and protective effects of silymarin on ischemia and reperfusion injury in the kidney tissues of rats. Int Urol Nephrol. 2008; 40(2):453-60. doi: 10.1007/S11255-008-9365-4.

27. Bayrak O, Turgut F, Karatas OF, Cimentepe E, Catal $\mathrm{F}$, Atis $\mathrm{O}$, et al. Oral beta-glucan protects kidney agaist ischemia/reperfusion injury in rats. Am J Nephrol. 2008; 28(2):190-6. doi: 10.1159/000 110087.

28. Yanarates O, Guven A, Sizian A, Uysal B, Akgul O, Ozcan A, et al. Ameliorative effects of proanthocyanidin on renal ischemia/reperfusion injury. Ren Fail. 2008; 30(9):931-8. doi: 10.180/088 60220802359410.

29. Aktoz T, Aydogdu N, Alagol B, Yalcin O, Huseyinova $\mathrm{G}$, Atakan $\mathrm{H}$. The protective effects of melatonin and vitamin $\mathrm{E}$ against renal ischemia-reperfusion injury in rats. Ren Fail. 2007; 29(5):535-42. doi: 10.180/08860220701391738.

30. Kirpatovskii VI, Golod EA, Nadtochii ON, Obukhova TV. Effects of alpha-tocopherol on partial functions of the ischemic kidney. Urologia. 2006; (5):80-4.

31. Korkmaz A, Kolankaya D. The protective effects of ascorbic acid against renal ischemia-reperfusion injury in male rats. Ren Fail. 2009; 31(1):36-43. doi: 10.180/0886022080254627.

32. Uysal F, Girgin FK, Tuzun S, Aldemir S, Sozmen EY. Effect of vitamin $E$ on antioxidant enzymes and nitric oxide in ischemia-reperfused kidney injury. Biochem Mol Biol Int. 1998; 44(6):1255-63. doi: 10.180/15216549800202352.

33. Shimizu MHM, Araujo M, Borges SMM, Tolosa EMC, Seguro AC. Exper Gerontol. 2004; 39(5):82530. doi: 10.1016/j.exger.2004.02.009.

34. Sener G, Tugtepe H, Yuksel M, Cetinel N, Yegen $B C$. Resveratrol improves ischemia/reperfusioninduced oxidative renal injury in rats. Arch Med Res. 2006; 37(7):822-9. doi:10.1016/j.arcmed. 2006.04.003.

35. Rah DK, Han HS, Baek HS, Hyon SH, Park BY, Park $J C$. Protection od rabbit kidney from ischemia/ reperfusion injury by gree tea polyphenol pretreatment. Arch Pharm Res. 2007; 30(11): 1447-54. doi: 10.1016/BF02977370.

36. Lee Jl, Son HY, Kim MC. Attenuation of ischemiareperfusion injury by ascorbic acid in the canine renal transplantation. J Vet Sci. 2006; 7(4):375-9.

37. Kuntscher V, Treska V, Racek J, Trefil L, Hes O. Does the administration of antioxidants as scavengers of reactive oxygen species in kidney transplantation really have sense? Bratisl Lek Listy. 2007; 108(9): 385-7.

Recebido em: 15/4/2008

Versão final reapresentada em: 22/7/2009

Aprovado em: 11/11/2009 